MATEC Web of Conferences 13,03001 (2014)

DOI: $10.1051 /$ matecconf/ 20141303001

(C) Owned by the authors, published by EDP Sciences, 2014

\title{
A Framework for Estimating Piping Reliability Subject to Corrosion Under Insulation
}

\author{
Ainul Akmar Mokhtar ${ }^{1, \mathrm{a}^{*},}$ Mokhtar Che Ismail ${ }^{2}$, Abu Bakar Zainordin ${ }^{3}$, Shahrullizam Shahid ${ }^{4}$ \\ ${ }^{1,2}$ Mechanical Engineering Department, Universiti Teknologi PETRONAS, Bandar Seri Iskandar, \\ 31750 Tronoh, Perak \\ ${ }^{3,4}$ PETRONAS Gas Berhad, Km 105, Jalan Kuala Terengganu-Kuantan, 24300 Kerteh, Terengganu
}

\begin{abstract}
Corrosion under insulation (CUI) is one of the serious damage mechanisms experienced by insulated piping systems. Optimizing the inspection schedule for insulated piping systems is a major challenge faced by inspection and corrosion engineers since CUI takes place beneath the insulation which makes the detection and prediction of the damage mechanism harder. In recent years, risk-based inspection (RBI) approach has been adopted to optimize CUI inspection plan. RBI approach is based on risk, a product of the likelihood of a failure and the consequence of such failure. The likelihood analysis usually follows either the qualitative or the semi-qualitative methods, thus precluding it to be used for quantitative risk assessment. This paper presents a framework for estimating quantitatively the likelihood of failure due to CUI based on the type of data available.
\end{abstract}

\section{Introduction}

For insulated piping systems, optimizing the inspection schedule is one of the major challenges faced by inspection engineers as well as corrosion engineers since the damage mechanisms take place beneath the insulation which makes it hardly to detect and to predict. One of the serious degradation mechanisms experienced by insulated piping systems is corrosion under insulation (CUI). According to the statistics given by Exxon Mobil Chemical in September 2003, the highest incidence of leaks in the refining and chemical industries is due to CUI and not to process corrosion which contributed up to $40 \%$ to $60 \%$ of piping maintenance costs [1].

In the last years, risk-based inspection (RBI) approach, which prioritizes the components for inspection based on the risk estimated, has been adopted to optimize the inspection plan. Risk is defined as the product of the likelihood of failure and the consequence of such failure. At present, the assessment of the likelihood of failure for CUI follows either the qualitative or the semi-qualitative methods, thus precluding it to be used for quantitative risk assessment. The difficulty in applying the quantitative model is due to lack of failure data as well as the wall thickness data where these types of data are typically used in the reliability analysis. Therefore, the objective of this paper is to propose a framework for quantitatively assessing the likelihood of CUI failure. 


\section{Methodology}

The study was driven by the type of data available for CUI where in this case the wall thickness data, which is typically used to assess corrosion failure, were found to be quite limited. Therefore, several mathematical models were explored to go well with the available data as shown in Figure 1. It can be seen that there are typically three types of CUI inspection data as follows:

1) Visual inspection data: This data were collected after insulation was removed, however, no wall thickness measurements were being taken. Data was recognized as either CUI was found or CUI was not found i.e. pass or fail data.

2) Wall thickness data: This data were collected after insulation was removed at the thickness measurement locations (TMLs).

3) Design and operating data: Examples of design and operating data collected are design pressure and temperature as well as operating pressure and temperature.

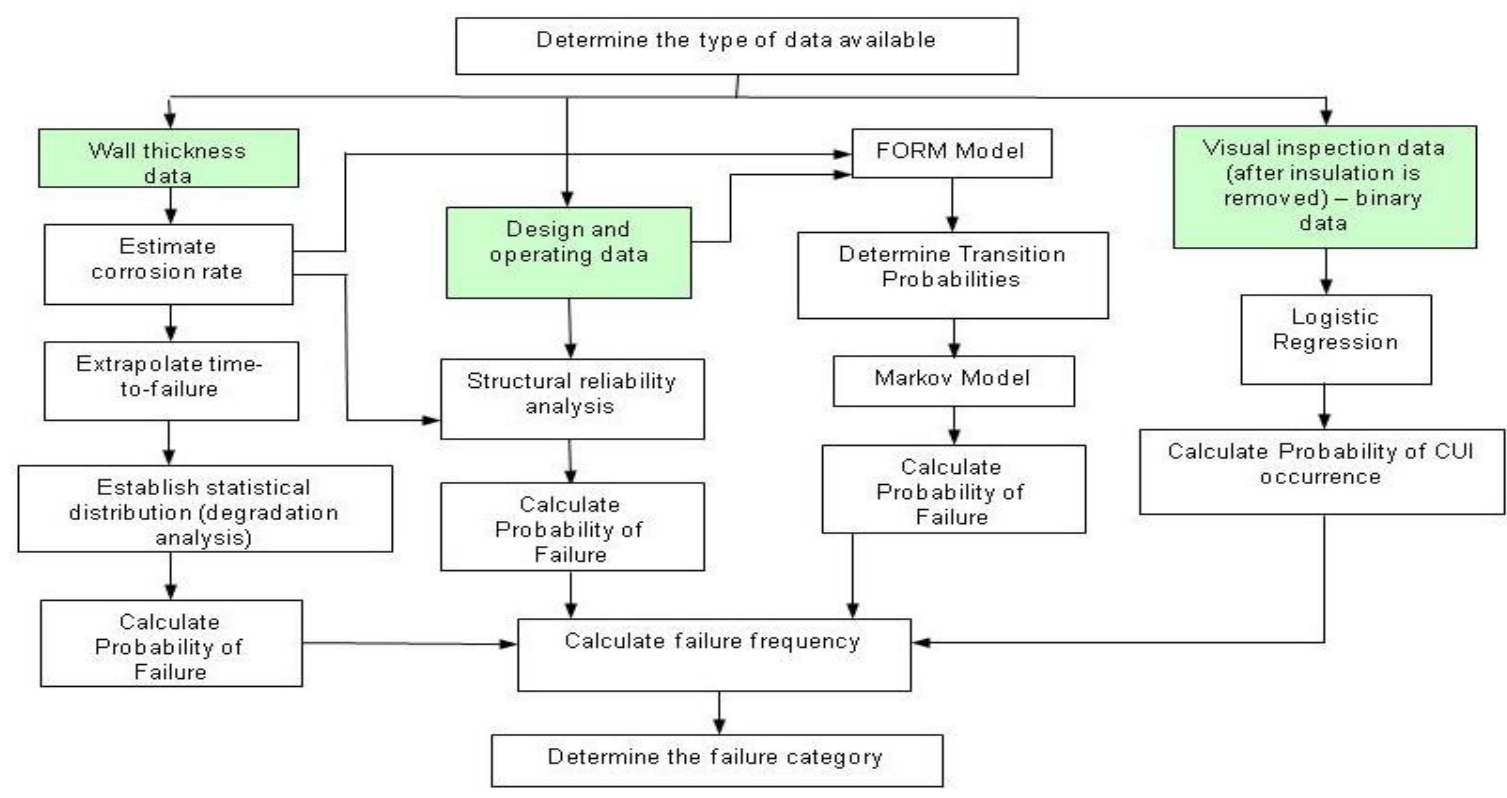

Figure 1. Proposed framework for assessing likelihood of CUI failure

Four models were proposed in the framework as follows. The next section will discuss the details of each model.

1) Logistic regression model: This model was applied based on the visual inspection data which can be treated as binary data $(1=$ CUI was found; $0=$ CUI was not found $)$.

2) Degradation analysis: This model was applied if the wall thickness data are available and the number of data is sufficient to carry out the degradation analysis. This model does not require the design and operating data.

3) Structural reliability analysis: This model was applied when the wall thickness data are limited and it took the advantage of having the design and operating data.

4) Continuous time Markov model: This model assumes the corrosion progresses through several states and in this study, the data required for this model was similar to the structural reliability model in determining the transition rates. 


\subsection{Logistic Regression Model}

Typically for CUI, the available data in CUI inspection reports is the result from inspection after insulation removal which is corrosion was found and treated, or corrosion was not seen. These types of data are classified as binary responses with 0 and 1 . Binary responses can be used to predict the probability of CUI occurrence by analysing binary data using logistic regression model [2]. The methods employed in an analysis using logistic regression follow the same general principles used in linear regression. However, there are two main differences in logistic regression compared to linear regression. The first difference is that in logistic regression, the response variables are categorical where the values are binary rather than continuous. Another major difference between logistic and linear regression is that the response variable is assumed to follow a binomial distribution rather than a normal distribution for response variable in linear regression. The logistic function, $f(z)$ that describes the mathematics behind this regression is as shown in Eq. (1). The logistic function $f(z)$ ranges between 0 and 1 .

$$
f(z)=\frac{1}{1+e^{-z}}
$$

From the logistic function, the logistic regression model is obtained through the parameter $z$ that can be written as the linear sum of the explanatory variables as shown in Eq. (2).

$$
z=\beta_{0}+\beta_{1} x_{1}+\beta_{2} x_{2}+\ldots+\beta_{n} x_{n}
$$

where $x_{1}, x_{2}, \ldots x_{n}$, are defined as the independent variables of interest and $\beta_{1}, \beta_{2} \ldots \beta_{n}$ are the coefficient representing unknown parameters. A regression can simultaneously handle both quantitative and qualitative explanatory variables. In this study, the quantitative variables are pipe age and operating temperature and the qualitative variable is type of insulation. Ref [3] showed how the model was applied for CUI cases.

\subsection{Degradation Analysis}

In a situation where failure data are scarce, degradation analysis is employed for the analysis of failure time distributions aiming to perceive the link between the degradation measurements and the failure time. For piping systems subject to corrosion, the degradation measurements are the wall thickness data, typically measured at the thickness measurement locations during each inspection period. The data are then fitted to an appropriate degradation models such as linear, exponential, power or logarithmic models. Degradation models vary markedly across the fields of reliability modelling. Using the degradation model, the "time-to-failure" is being assessed where in this context, the use of the term failure is defined as when the pipe wall thickness reaches the minimum wall thickness allowed. In other words, the failure can be defined as when the corrosion defect reaches a prespecified threshold value. The "time-to-failure" data are then fitted to an appropriate distribution and the likelihood of failure can be estimated. Ref [4] shows the details of how the analysis is being done.

\subsection{Structural Reliability Analysis}

Structural reliability analysis model is proposed when the wall thickness data are limited and it takes the advantage of having the design and operating data. This probabilistic approach is capable in incorporating the uncertainties in the reliability assessment. In assessing the reliability of structures, the following steps are typically followed:

1. To identify the limit-state function that defines the failure.

2. To assess the variability that lies in the input variables of the limit-state function. 
A limit state function needs to be defined where this function expresses the criterion for failure of the pipe. It is common for the limit state function to be the difference between resistance and the load. For corrosion failure in pipe, the thinning model proposed by Khan et al. [5] was used as the limit state function in this study and the function can be defined as Eq. (3).

$$
g(x)=R-S t=S\left(1-\frac{C R \times T}{t}\right)-\frac{P D}{2 t}
$$

where $R$ is material resistance (in $\mathrm{MPa}$ ), $S t$ is the applied stress (in MPa), $S$ is material yield strength (in $\mathrm{MPa}$ ), $C R$ is corrosion rate (in $\mathrm{mm} / \mathrm{yr}$ ), $P$ is operating pressure (in $\mathrm{MPa}$ ), $D$ is the diameter of the component (in $\mathrm{mm}$ ), $t$ is the material thickness (in $\mathrm{mm}$ ) and $T$ is number of years in service (in year). The variability of the random variables $S, C R, P, D$ and $t$ is reviewed and estimation of their probability distributions and their parameters are made. Ref $[4,6]$ shows the details of how the analysis is being done.

\subsection{Continuous-Time Markov Model}

A three-state Markov model for general wall thinning proposed by Fleming [6] is employed in this study, as shown in Figure 2. The state transition rates are denoted by $\phi, \lambda$ and $\omega$. Fleming defined the three states; State 1 (no detectable damage), State 2 (detectable flaw (wall thinning) and State 3 (failure i.e. leak or rupture). In this model, the occurrence of leaks is defined as a failure which does not mean there are actual leaks but rather the thickness of wall has reached the minimum wall thickness specified. The definitions given for each state in Fleming model were very general. Therefore, this study proposed the definition shown in Table 1. The definition of states can be based on the depth of corrosion defects as shown in Figure 3.

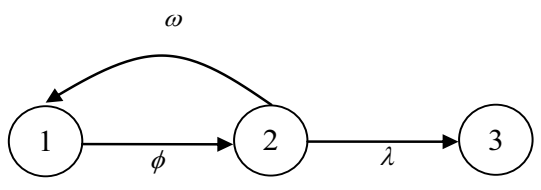

Figure 1. Three state continuous time Markov model [6]

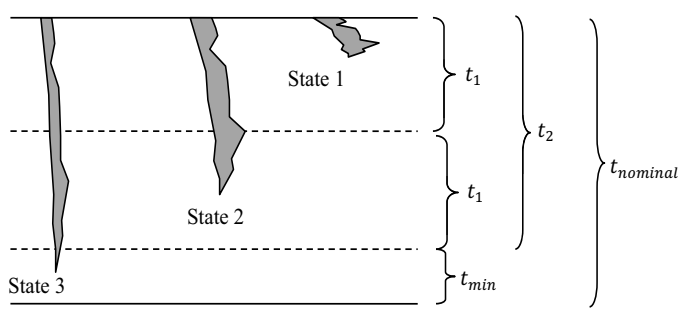

Figure 2. Illustration for the limit-state functions used to estimate the transition rates

Table 1: Description for the three-state Markov model

\begin{tabular}{|c|c|}
\hline State & Definition \\
\hline 1 & $d$ less than $t_{1}$ \\
\hline 2 & $d$ is between $t_{1}$ and $t_{2}$ \\
\hline 3 & $d$ is more than $t_{2}$ \\
\hline
\end{tabular}


Note: $d$ is the depth of corrosion, $t_{1}=\left(t_{\text {nomin } a l}-t_{\min }\right)-\left(\frac{\left(t_{\text {nomin } a l}-t_{\min }\right)}{2}\right), t_{2}=t_{\text {nomin } a l}-t_{\min }$

\section{Conclusions}

This paper outlines the four proposed models in assessing quantitatively the likelihood of failure due to CUI based on the types of data available. The models are logistic regression model, degradation analysis, structural reliability analysis and continuous time Markov model. The followings are the results of the study:

$>$ A good quantitative model that can be applied with the current CUI data is the structural reliability analysis model where the reliability calculation is based on the design and operating data as well as the wall thickness data to estimate the corrosion rate. However, in the case where the wall thickness data is abundant, the degradation analysis is proposed to be used.

$>$ The Markov model seems to be a potential model in the future. To adopt and apply it successfully, then several steps have to be put in place such as:

- The number of states and the definition of each state have to be developed properly by having consensus from experts/plant experience because these two factors give a big impact to the likelihood of failure calculation.

- The method to estimate the transition rates should be established. The transition rate estimated for the Markov model developed in this study was based on the design data, not from the actual plant data.

$>$ Using logistic regression model, the relationship between the binary outcomes and the factors contributed to CUI was modelled mathematically. This model demonstrated that without the wall thickness data typically used to analyse corrosion problem, the probability of CUI occurrence can still be generated. However, the results do not tell the severity of CUI in term of depth of corrosion. For that reason, the results generated cannot be employed in the current quantitative risk assessment. The model can only be a guideline for inspection planning purpose i.e. which insulated pipe to be checked based on given age, operating temperature and insulation type.

\section{References}

1. Corrosion under insulation (http://www.nansulate.com/pdf/CUI_nanotechnology_solution.pdf, n.d)

2. D.W. Hosmer, S. Lemeshow. Applied Logistic Regression (John Wiley \& Sons, New York, 1989)

3. A.A. Mokhtar, N. Saari, M. Che Ismail in 8th World Congress on Eng Asset Management \& 3rd Int Conf on Utility Management \& Safety (2013)

4. A.A. Mokhtar, M. Che Ismail, M. Muhammad, Int J Eng Tech, 9, 10, 99-104 (2009).

5. F.I. Khan, M.M. Haddara, S.K. Bhattacharya. Risk Anal, 26, 1, 203-221 (2006).

6. A.A. Mokhtar, M. Che Ismail. J Appl Sci. doi:10.3923/jas.2011 (2011).

7. K. Fleming. Reliab Eng Syst Safe, 83, 27-45 (2004). 